\title{
On the Patterson-Sullivan measure of some discrete group of isometries
}

\author{
Peigné Marc \\ Université de Tours I, Parc de Grandmont \\ 37200 Tours \\ e.mail : peigne@univ-tours.fr
}

The recent development of the study of discrete conformal groups $\Gamma$ acting on the $d+1$ dimensional ball $\mathbb{B}^{d+1}$ and the associated dynamics is closely related to ideas considered by Poincaré himself, who interpretated the interior of $\mathbb{B}^{d+1}$ and its group of conformal transformations as a model of the $d+1$-dimensional hyperbolic space $\mathbb{H}^{d+1}$. One of the main tools he introduced is the series $\sum_{\gamma \in \Gamma}\left|\gamma^{\prime} \mathbf{x}\right|^{s}$ of the group $\Gamma$, where $\left|\gamma^{\prime} \mathbf{x}\right|$ is the linear distortion of the Euclidean metric by the conformal transformation $\gamma$ and $\mathbf{x}$ lies in the interior of $\mathbb{B}^{d+1}$; in particular, the critical exponent $\delta_{\Gamma}$ of this series plays a central role in this theory, appearing in other guises for certain groups, as for example the Hausdorff dimension of the limit set or the topological entropy of the geodesic flow.

At the end of the seventies, S. J. Patterson proposed, for any discrete conformal group $\Gamma$, a geometrical construction of a family $\left(\sigma_{\mathbf{x}}\right)_{\mathbf{x} \in \mathbb{B}^{d+1}}$ of measures on the $d$-dimensional unit sphere $\mathbb{S}^{d}$ which are $\delta_{\Gamma^{-}}$conformal. Just as the Lebesgue measure on $\mathbb{S}^{d}$ is associated to an invariant measure for the geodesic flow on the unit tangent bundle of $\mathbb{H}^{d+1}$, such family $\left(\sigma_{\mathbf{x}}\right)$ determines an invariant measure $\mu^{\sigma}$ for the geodesic flow on the unit tangent bundle of the manifold $\mathbb{H}^{d+1} / \Gamma$. The dichotomy $\sum_{\gamma \in \Gamma}\left|\gamma^{\prime} \mathbf{x}\right|^{\delta_{\Gamma}}$ finite or infinite is thus equivalent to the Hopf dichotomy, namely complete nonrecurrence, or conservativity and ergodicity of the geodesic flow with respect to $\mu^{\sigma}[13]$. When the manifold $\mathbb{H}^{d+1} / \Gamma$ is compact or has finite volume, the Poincaré series diverges at its critical exponent, the measure $\sigma_{\text {o }}$ coincides with the Lebesgue measure on $\mathbb{S}^{d}$ and $\mu^{\sigma}$ is nothing else than the Liouville measure on the unit tangent bundle of $\mathbb{H}^{d+1} / \Gamma$; in particular, $\mu^{\sigma}$ is finite since $\Gamma$ is a lattice. More generally, if $\Gamma$ is geometrically finite, its Poincaré series diverges at $\delta_{\Gamma}$ and the measure $\mu^{\sigma}$ is also finite [13].

On the other hand, there is an interrelation between the Poincaré exponent $\delta_{\Gamma}$ and the square root of the lowest eigenvalue of the hyperbolic Laplacian $\Delta_{H}$ on $\mathbb{H}^{d+1} / \Gamma$; in distinct terms, the function $\Phi_{\sigma}: \mathbf{x} \mapsto \sigma_{\mathbf{x}}\left(\mathbb{S}^{d}\right)$ is a positive and $\Gamma$-invariant $\delta_{\Gamma}\left(\delta_{\Gamma}-d\right)$-eigenfunction of $\Delta_{H}$. Furthermore, if $\mu^{\sigma}$ is finite, the function $\Phi_{\sigma}$ belongs to $\mathbb{L}^{2}$ (for the volume form) of $I H^{d+1} / \Gamma$ if and only if $\delta_{\Gamma}>d / 2$; nevertheless, when $\delta_{\Gamma} \leq d / 2$, the function $\Phi_{\sigma}$ belongs to the space $\mathbb{L}^{2}$ of some relevant part of $\mathbb{H}^{d+1} / \Gamma$, namely any $\epsilon$-neigbourhood $N^{\epsilon}(\Gamma)$ of 
its Nielsen core $N(\Gamma)$.

In [14], D. Sullivan asked whether there are others groups besides geometrically finite ones where $\Phi_{\sigma}$ belongs to $\mathbb{L}^{2}\left(\mathbb{H}^{d+1} / \Gamma\right)$ when $\delta_{\Gamma}>d / 2$ or to $\mathbb{L}^{2}\left(N^{\epsilon}(\Gamma)\right)$ when $\delta_{\Gamma} \leq d / 2$. Since the square integrability of $\Phi_{\sigma}$ on $N^{\epsilon}(\Gamma)$ is equivalent to the finiteness of $\mu^{\sigma}$, D. Sullivan's problem may be formulated as follows

Does there exist non geometrically finite groups with associated Patterson-Sullivan measure $\mu^{\sigma}$ of finite total mass?

In this paper, we give a positive answer to this question and describe a large class of such non geometrically finite groups. This is of interest since recent results by Th. Roblin on orbital functions of general discrete groups $\Gamma$ have been obtained under the sole condition of finiteness of $\mu^{\sigma}[11]$.

\section{Notations and main results}

The unit ball model of the hyperbolic space $\mathbb{H}^{d+1}$ is $\mathbb{B}^{d+1}=\left\{\mathbf{x} \in \mathbb{R}^{d+1} /\|\mathbf{x}\|<1\right\}$ endowed with the hyperbolic distance (.,.). A Kleinian group $\Gamma$ is a discrete torsion free group of orientation-preserving isometries of $\mathbb{H}^{d+1}$. It acts by conformal transformations on the sphere $\mathbb{S}^{d}$ endowed with the euclidean metric |.|. The limit set $\Lambda_{\Gamma}$ of $\Gamma$ is the set of accumulation points of any $\Gamma$-orbit. We will assume that $\Gamma$ is non elementary which means that $\Lambda_{\Gamma}$ contains infinitely many points.

Let $\mathbf{x}$ and $\mathbf{y}$ be two points in $\mathbb{H}^{d+1}$ and $s \in \mathbb{R}^{+}$. The Poincaré series of $\Gamma$ is defined by $P_{\Gamma}(\mathbf{x}, \mathbf{y}, s)=\sum_{\gamma \in \Gamma} e^{-s(\mathbf{x}, \gamma \cdot \mathbf{y})}$. The Poincaré exponent $\delta_{\Gamma}$ of $\Gamma$ is the infimum of the set of $s$ such that $P_{\Gamma}(\mathbf{x}, \mathbf{y}, s)$ is finite; it does not depend on $\mathbf{x}$ and $\mathbf{y}$. One says that $\Gamma$ is convergent (resp. divergent) if $P_{\Gamma}\left(\mathbf{x}, \mathbf{y}, \delta_{\Gamma}\right)<+\infty\left(\operatorname{resp} . P_{\Gamma}\left(\mathbf{x}, \mathbf{y}, \delta_{\Gamma}\right)=+\infty\right)$.

Quotienting the hyperbolic space by a Kleinian group $\Gamma$ leads to a hyperbolic manifold $M=\mathbb{H}^{d+1} / \Gamma$. The Nielsen core $N(\Gamma)$ of $M$ is the convex submanifold of $M$ obtained by quotienting by $\Gamma$ the convex hull of $\Lambda_{\Gamma}$. One says that $\Gamma$ is geometrically finite when some (and so any) $\epsilon$ - neighbourhood $N^{\epsilon}(\Gamma)$ of $N(\Gamma)$ in $M$ has finite volume; if $d>2$ the definition of a geometrically finite group with torsion requires finitely generatedness (see $[7])$.

A classical way to decide whether or not a group $\Gamma$ is divergent is to consider a Patterson

measure that is to say a cluster point for the weak convergence on $\overline{H^{d+1}}$ of a family of measures $\left(\sigma_{\mathbf{x}, \mathbf{y}, s}\right)_{s>\delta_{\Gamma}}$ supported by the orbit $\Gamma . \mathbf{y}$ seen from the point $\mathbf{x}$. By TsujiHopf-Sullivan's theorem [13], the group $\Gamma$ is divergent if and only if these measures are supported by the radial limit set of $\Gamma$, which is the set of points $\xi \in \Lambda_{\Gamma}$ for which there exist infinitely many distinct points of the $\Gamma$-orbit of $\mathbf{o}$ at a bounded distance of the geodesic ray $[\mathbf{o}, \xi)$. As observed by D. Sullivan [12], a Patterson measure can be used to construct a measure $\mu^{\sigma}$ on $T^{1} M$, called a Patterson -Sullivan measure, which is invariant under the action of the geodesic flow and supported by its non-wandering set. When $\Gamma$ is geometrically finite, it is divergent and $\mu^{\sigma}$ is finite [13]. 
We will say that two Kleinian groups $G$ and $H$ are in Schottky position if there exist disjoint closed sets $F_{G}$ and $F_{H}$ in $\mathbb{S}^{d}$ such that

$$
G^{*}\left(\mathbb{S}^{d}-F_{G}\right) \subset F_{G} \text { and } H^{*}\left(\mathbb{S}^{d}-F_{H}\right) \subset F_{H} .
$$

where $G^{*}=G-\{I d\}$ and $H^{*}=H-\{I d\}$. Note that $\left(F_{G}, F_{H}\right)$ is a proper interactive pair of sets (see [8] VII, A.6 and A.9); the Klein combination theorem implies that the group $\Gamma$ generated by $G$ and $H$ is equal to the free product $G * H$. We have the

Theorem A - Let $\Gamma=G * H$ be the free product of two Kleinian groups in Schottky position. If $\delta_{\Gamma}>\max \left(\delta_{G}, \delta_{H}\right)$ then $\Gamma$ is divergent and its Patterson-Sullivan measure $\mu^{\sigma}$ is finite.

Remark 1- By corollary 1 in [10] one has $\delta_{\Gamma}>\max \left(\delta_{G}, \delta_{H}\right)$ as soon as the subgroup $G$ or $H$ of maximal critical exponent is divergent (see also ([6], Theorem 1) and more recently ([5], Proposition 2) for similar statements).

The main consequence of Theorem $\mathrm{A}$ is the following :

Corollary 1 -There exist non geometrically finite groups $\Gamma$ with finite Patterson-Sullivan measure.

More precisely, there exist non geometrically finite groups $\Gamma$ with Poincaré exponent $\delta_{\Gamma}>$ $d / 2$ (resp. $\delta_{\Gamma} \leq d / 2$ ) for which the positive eigenfunction $\Phi_{\sigma}$ belongs to $\mathbb{L}^{2}\left(\mathbb{H}^{d+1} / \Gamma\right)$ (resp. to $\mathbb{L}^{2}\left(N^{\epsilon}(\Gamma)\right)$.

Recently and independently, A. Ancona obtained the same result by methods based on potential theory [1].

On the hyperbolic plane $\mathbb{H}^{2}$, a discrete group of isometries is geometrically finite if and only if it is finitely generated ; thus, our examples will be infinitely generated for $d=1$. When $d \geq 2$, L. Bers proved that there exist finitely generated Kleinian groups which are not geometrically finite [2]; in this case, one can thus specify D. Sullivan's problem and ask whether there are finitely generated groups which are not geometrically finite and whose Patterson-Sullivan measure is finite. If $d=2$, the Ahlfors conjecture states that the limit set of a finitely generated Kleinian group $\Gamma$ is either the whole sphere or has zero area. A recent work by C.J. Bishop and P.W. Jones [3] shows that this conjecture would imply that $\delta_{\Gamma}=2$ and $\mu^{\sigma}$ is infinite when $\Gamma$ is a non geometrically finite group of finite type. In higher dimension we have the following result :

Corollary 2 - If $d \geq 3$ there exist non geometrically finite groups of finite type whose Patterson-Sullivan measure is finite.

Acknowledgements : : We thank M. Babillot for her warm encouragements all along this work and for pointing out to us the reference [14]. We thank J.P. Otal for helpfull conversations about the geometry of Kleinian groups, E. Lesigne for some elegant remarks in ergodic theory and the referee for a number of helpfull comments and references. 


\section{Coding the limit set of Schottky product groups}

It has been known for a long time that the limit set (minus a countable subset) of a classical Schottky group $\Gamma$ can be identified with a subshift of finite type $\Sigma_{\mathcal{A}}^{+} \subset \mathcal{A}^{\mathbb{N}}$ where $\mathcal{A}=\left\{a_{1}, \ldots, a_{n}\right\}$. Furthermore, the action of $\Gamma$ on this large part of its limit set is orbit equivalent to the one of the shift operator on $\Sigma_{\mathcal{A}}^{+}$. In this section we shall extend this construction to general Schottky product groups.

We consider here two non elementary Kleinian groups $G$ and $H$ in Schottky position and $F_{G}, F_{H}$ the associated closed subsets of $\mathbb{S}^{d}$ satisfying condition $(S)$. The group $\Gamma$ generated by $G$ and $H$ is Kleinian and is the free product of $G$ and $H$ ([8], theorem A.13)). Any element of $\Gamma^{*}$ has a unique normal form $\gamma=a_{1} \ldots a_{n}$ where either every $a_{k}$ with even $k$ lies in $G^{*}$ and every $a_{k}$ with odd $k$ lies in $H^{*}$ or vice versa ; the integer $n$ is called the length of the normal form of $\gamma$ and the elements $a_{1}$ and $a_{n}$ are respectively the first letter and last letter of $\gamma$.

The conformal factor of $\gamma \in \Gamma$ at the point $\xi \in \mathbb{S}^{d}$ is $\left|\gamma^{\prime} \xi\right|=e^{B_{\xi}\left(\gamma^{-1} \mathbf{o}, \mathbf{o}\right)}$ where, for any $\mathbf{y}, \mathbf{z} \in \mathbb{H}^{d+1}$ the quantity $B_{\xi}(\mathbf{y}, \mathbf{z})=\lim _{\mathbf{x} \rightarrow \xi}(\mathbf{y}, \mathbf{x})-(\mathbf{z}, \mathbf{x})$ represents the algebraic distance between the two horospheres centered at $\xi$ and passing trought $\mathbf{y}$ and $\mathbf{z}$. Furthermore for any $\xi, \eta \in \mathbb{S}^{d}$ one has $|\gamma \cdot \xi-\gamma \cdot \eta|^{2}=\left|\gamma^{\prime} \xi\right| \cdot\left|\gamma^{\prime} \eta\right| \cdot|\xi-\eta|^{2}$.

The following lemma describes the behavior on $F_{G} \cup F_{H}$ of the conformal factors of the elements of $\Gamma$.

Lemma 1 - i) The quantity $\left|B_{\xi}\left(a^{-1} \cdot \mathbf{o}, \mathbf{o}\right)-(\mathbf{o}, a \cdot \mathbf{o})\right|$ is bounded uniformly in $\xi \in F_{H}$ and $a \in G^{*}$ (resp. in $\xi \in F_{G}$ and $a \in H$ ).

ii) There exists $n_{0} \geq 1$ such that the quantity $B_{\xi}\left(\gamma^{-1} \cdot \mathbf{o}, \mathbf{o}\right)$ is bounded from below by 1 uniformly in $\xi \in F_{H}$ and $\gamma \in \Gamma$ with length of normal form $\geq n_{0}$ and last letter in $G^{*}$ (resp. $\xi \in F_{G}$ and $\gamma \in \Gamma$ with last letter in $H^{*}$ ).

Note that the second statement does not hold in the presence of torsion.

Proof- The set $\left\{a^{-1} \cdot \mathbf{o} / a \in G\right\}$ accumulates in $F_{G}$; since the visibility angle between $F_{G}$ and $F_{H}$ is bounded from below, the quantity $B_{\xi}\left(a^{-1} \cdot \mathbf{o}, \mathbf{o}\right)-(\mathbf{o}, a \cdot \mathbf{o})$ is thus bounded uniformly in $\xi \in F_{H}$ and $a \in G^{*}$. The first assertion of the Lemma follows letting $(\mathbf{o}, \gamma \cdot \mathbf{o}) \rightarrow+\infty$.

To prove the second assertion, one remarks that $\left\{\gamma^{-1} \cdot \mathbf{o} / \gamma \in \Gamma\right.$ with last letter in $\left.G^{*}\right\}$ accumulates in $F_{G}$. The quantity $B_{\xi}\left(\gamma^{-1} \cdot \mathbf{o}, \mathbf{o}\right)-(\mathbf{o}, \gamma \cdot \mathbf{o})$ is thus bounded uniformly in $\xi \in F_{H}$ and $\gamma \in \Gamma$ with last letter in $G^{*}$; one thus has $B_{\xi}\left(\gamma^{-1} \cdot \mathbf{o}, \mathbf{o}\right) \geq 1$ for all $\xi \in F_{H}$ and all but finitely many $\gamma$ with last letter in $G^{*} . \square$

Lemma 1 implies that there exist $0<r<1$ and $C>0$ such that for all $\xi \in F_{H}$ (resp. $\left.\xi \in F_{G}\right)$ and all $\gamma=a_{1} \cdots a_{n} \in \Gamma$ with $a_{n} \in G^{*}\left(\right.$ resp. $\left.a_{n} \in H^{*}\right)$, one has

$$
\text { (*) } \quad\left|\gamma^{\prime} \xi\right| \leq C r^{n} \text {. }
$$

Proposition 1 - Denote by $\Sigma^{+}$the set of sequences $\mathbf{a}=\left(a_{n}\right)_{n \geq 1}$ for which each letter $a_{n}$ belongs to the alphabet $G^{*} \cup H^{*}$ and such that no two consecutive letters belong to the 
same group. Fix a point $\xi_{0}$ in $\mathbb{S}^{d}-\left(F_{G} \cup F_{H}\right)$. Then

a) For any $\mathbf{a}=\left(a_{n}\right) \in \Sigma^{+}$, the sequence $\left(a_{1} \cdots a_{n} \cdot \xi_{0}\right)_{n \geq 1}$ converges to a point $\pi(\mathbf{a})$ which belongs to the limit set of $\Gamma$ and does not depend on $\xi_{0} \in \mathbb{S}^{d}-\left(F_{G} \cup F_{H}\right)$.

b) The $\operatorname{map} \pi: \Sigma^{+} \rightarrow \Lambda_{\Gamma}$ is one-to-one.

c) The set $\Lambda^{0}=\pi\left(\Sigma^{+}\right)$is included in the radial limit set of $\Gamma$.

d) The set $\Lambda_{\Gamma}-\Lambda^{0}$ is equal to the $\Gamma$-orbit of $\Lambda_{G} \cup \Lambda_{H}$.

Proof. Fix $\mathbf{a} \in \Sigma^{+}$and, for $n, p \geq 1$, set $\xi_{n, p}=a_{n+1} \cdots a_{n+p} \cdot \xi_{0}$; by inequality $(*)$ one has $\left|a_{1} \cdots a_{n} \cdot \xi_{0}-a_{1} \cdots a_{n+p} \cdot \xi_{0}\right| \leq 2 C r^{n-1}$ and so $\left(a_{1} \cdots a_{n} \cdot \xi_{0}\right)_{n \geq 1}$ is a Cauchy sequence ; a similar argument proves that its limit $\pi(\mathbf{a})$ does not depend on $\xi_{0}$.

To prove $b$ ), we consider two sequences $\mathbf{a}$ and $\mathbf{b}$ which differ from the first time at time $n$. Set $\xi_{n}=\lim _{p \rightarrow+\infty} a_{n} \cdots a_{n+p} \cdot \xi_{0}$ and $\xi_{n}^{\prime}=\lim _{p \rightarrow+\infty} b_{n} \cdots b_{n+p} \cdot \xi_{0}$. If $a_{n}$ and $b_{n}$ do not belong to the same set $G^{*}$ or $H^{*}$, the points $\xi_{n}$ and $\xi_{n}^{\prime}$ do not belong to the same set $F_{G}$ or $F_{H}$; otherwise, the same property holds for the points $a_{n}^{-1} \cdot \xi_{n}$ and $a_{n}^{-1} \cdot \xi_{n}^{\prime}$ since $a_{n} \neq b_{n}$. In all the cases $\pi(\mathbf{a})$ and $\pi(\mathbf{b})$ are distinct.

To prove c), we use the fact that a point $\xi \in \Lambda_{\Gamma}$ is radial if and only if there exists a sequence $\left(\gamma_{k}\right)$ of distinct elements in $\Gamma$ such that for any $\eta \in \Lambda_{\Gamma}-\{\xi\}$ the sequence $\left(\left(\gamma_{k} \cdot \xi, \gamma_{k} \cdot \eta\right)\right)_{k}$ belongs to some compact subset of the complement of the diagonal in $\Lambda_{\Gamma} \times \Lambda_{\Gamma}$ [4]. Actually, fix $\xi=\pi(\mathbf{a})$ with $\mathbf{a}=\left(a_{n}\right)$ and $a_{1} \in G^{*}$ and set $\gamma_{k}=a_{k}^{-1} \cdots a_{1}^{-1}$. The point $\gamma_{2 k} \cdot \xi$ belongs to $F_{G}$ and for any $\eta \in \Lambda_{\Gamma}-\{\xi\}$ and $k$ large enough, the point $\gamma_{2 k} \cdot \eta$ belongs to $F_{H}$.

Let us now prove d). Fix $\xi \in \Lambda_{\Gamma} \cap F_{G}$. Assume first that for all $g \in G$ the point $g^{-1} \cdot \xi$ belongs to $F_{G}$. Since $\xi \in \Lambda_{\Gamma}$ there exists a sequence $\left(\gamma_{k}\right)_{k}$ in $\Gamma$ such that $\xi=\lim _{k \rightarrow+\infty} \gamma_{k} \cdot \xi_{0}$; for $k$ large enough, the first letter $\alpha_{k}$ of $\gamma_{k}$ belongs to $G^{*}$. One can thus extract a subsequence of $\left(\gamma_{k}\right)$ (also denoted $\left(\gamma_{k}\right)$ ) such that the $\alpha_{k}$ are all distinct (otherwise, there would exist $\alpha \in G$ such that $\alpha_{k}=\alpha$ infinitely often and the point $\xi$ would belong to $\alpha\left(F_{H}\right)$ which contradicts the hypothesis) ; without loss of generality, setting $\beta_{k}=\alpha_{k}^{-1} \gamma_{k}$, one may assume that $\left(\beta_{k} \cdot \xi_{0}\right)_{k}$ converges to some $\eta_{0} \in F_{H}$. It follows $\xi=\lim _{k \rightarrow+\infty} \alpha_{k} \beta_{k} \cdot \xi_{0}=\lim _{k \rightarrow+\infty} \alpha_{k} \cdot \eta_{0}$ which proves that $\xi$ belongs to $\Lambda_{G}$.

Assume now that there exists $g \in G$ such that $g^{-1} \cdot \xi$ belongs to $F_{H}$; note that such a $g$ is unique when it exists, one thus sets $a_{1}=g$ and one applies the above discussion to the point $a_{1}^{-1} \cdot \xi$. When $\xi \notin \Gamma \cdot\left(\Lambda_{G} \cup \Lambda_{H}\right)$ one may construct a sequence $\mathbf{a}=\left(a_{n}\right) \in \Sigma^{+}$such that $\xi=\lim _{n \rightarrow+\infty} a_{1} \cdots a_{n} \cdot \xi_{0}$ and so $\xi \in \Lambda^{0}=\pi\left(\Sigma^{+}\right)$.

We now explain how to code the geodesic flow restricted to some particular subset of its non-wandering set. For a unit vector $v=\left(\mathbf{v}_{\mathbf{0}}, \vec{v}\right)$ in the unit tangent bundle of the hyperbolic space $T^{1} \mathbb{H}^{d+1}$, we let $v_{-\infty}$ and $v_{+\infty}$ be the endpoints on $\mathbb{S}^{d}$ of the unique geodesic passing throught $v$. One associates to $v$ the triplet $\left(v_{-\infty}, v_{+\infty}, r\right)$ where $r=B_{v_{+\infty}}\left(\mathbf{o}, \mathbf{v}_{\mathbf{0}}\right)$ and thus identifies $T^{1} \mathbb{H}^{d+1}$ with the set $\left(\mathbb{S}^{d} \times \mathbb{S}^{d}-\right.$ diagonal $) \times \mathbb{R}$ ; the geodesic flow $\left(\tilde{g}_{t}\right)$ on $T^{1} \mathbb{H}^{d+1}$ acts by translation along the third coordinate : $\tilde{g}_{t}\left(\xi^{-}, \xi^{+}, r\right)=\left(\xi^{-}, \xi^{+}, r+t\right)$ and the action of $\gamma \in \Gamma$ on $T^{1} H^{d+1}$ is given by

$$
\gamma\left(\xi^{-}, \xi^{+}, r\right)=\left(\gamma \cdot \xi^{-}, \gamma \cdot \xi^{+}, r-B_{\xi^{+}}\left(\mathbf{o}, \gamma^{-1} \cdot \mathbf{o}\right)\right)
$$


The action of $\left(\tilde{g}_{t}\right)$ commutes with the action of $\Gamma$ and induces the geodesic flow $\left(g_{t}\right)$ on $T^{1} M$. The subset $\left(\Lambda_{\Gamma} \times \Lambda_{\Gamma}-\right.$ diagonal $) \times \mathbb{R}$ of $T^{1} \mathbb{H}^{d+1}$ is both invariant under $\Gamma$ and $\left(\tilde{g}_{t}\right)$ and its projection on $T^{1} M$ coincides with the non-wandering set of the geodesic flow. Using the coding of the set $\Lambda^{0}$, we introduce in a natural way a $\left(g_{t}\right)$-invariant subset of $\left(\Lambda_{\Gamma} \times \Lambda_{\Gamma}-\right.$ diagonal $) \times \mathbb{R} / \Gamma$.

Proposition 2 - Let $\Sigma$ be the set of double sided sequences $\mathbf{a}=\left(a_{n}\right)_{n \in \mathbb{Z}}$ for which each letter $a_{n}$ belongs to $G^{*} \cup H^{*}$ and no two consecutive letters belong to the same group . Let $\pi$ be the map from $\Sigma$ to $\Lambda_{\Gamma} \times \Lambda_{\Gamma}$ - diagonal defined by

$$
\pi(\mathbf{a})=\left(\lim _{n \rightarrow+\infty} a_{0}^{-1} \cdots a_{-n}^{-1} \cdot \mathbf{o}, \lim _{n \rightarrow+\infty} a_{1} \cdots a_{n} \cdot \mathbf{o}\right) .
$$

Let $T$ be the invertible map on $\mathcal{D}^{0}=\pi(\Sigma)$ induced by the shift operator on $\Sigma$ and set $f\left(\xi^{+}\right)=-B_{\xi^{+}}\left(\mathbf{o}, a_{1} \cdot \mathbf{o}\right)$ where $a_{1}$ is the first letter of $\xi^{+}$. Then

a) The action of $\Gamma$ on $\Lambda^{0} \times \Lambda^{0}$ - diagonal is orbit equivalent with the action of $T$ on $\mathcal{D}^{0}=\pi(\Sigma)$.

b) The restriction of the geodesic flow $\left(g_{t}\right)$ to the set $\left(\Lambda^{0} \times \Lambda^{0}-\right.$ diagonal $) \times \mathbb{R} / \Gamma$ can be represented as a special flow constructed from the automorphism $T$ on $\mathcal{D}^{0}$ and the ceiling function $f$.

Proof- Fix $\xi^{-}=\pi(\mathbf{a})$ and $\xi^{+}=\pi(\mathbf{b})$ where $\mathbf{a}$ and $\mathbf{b}$ are two distinct sequences in $\Sigma^{+}$, and assume that $\mathbf{a}$ and $\mathbf{b}$ differ from the first time at time $n$; the point $\left(b_{1} \cdots b_{n-1}\right)^{-1} \cdot\left(\xi^{-}, \xi^{+}\right)$ belongs to $\mathcal{D}^{0}$ which proves that $\mathcal{D}^{0}$ is a section for the action of $\Gamma$. Furthermore if $\left(\xi^{-}, \xi^{+}\right) \in \mathcal{D}^{0}$ and $\gamma \in \Gamma$ one has $\gamma \cdot\left(\xi^{-}, \xi^{+}\right) \in \mathcal{D}^{0}$ if and only if $\gamma=\left(a_{1} \cdots a_{k}\right)^{-1}$ or $\gamma=\left(b_{1} \cdots b_{k}\right)^{-1}$ for some $k \geq 0$; in the first case, $\gamma \cdot\left(\xi^{-}, \xi^{+}\right)=T^{-k}\left(\xi^{-}, \xi^{+}\right)$and in the second case $\gamma \cdot\left(\xi^{-}, \xi^{+}\right)=T^{k}\left(\xi^{-}, \xi^{+}\right)$. The action of $\Gamma$ on $\left(\Lambda^{0} \times \Lambda^{0}-\right.$ diagonal $) \times \mathbb{R}$ is thus orbit-equivalent with the action of the transformation $T_{f}$ on $\mathcal{D}^{0} \times \mathbb{R}$ defined by

$$
T_{f}\left(\xi^{-}, \xi^{+}, r\right)=a^{-1} \cdot\left(\xi^{-}, \xi^{+}, r\right)=\left(a^{-1} \cdot \xi^{-}, a^{-1} \cdot \xi^{+}, r+f\left(\xi^{+}\right)\right) .
$$

This achieves the proof.

\section{The Patterson measure}

Using Proposition 1, we will prove the following

Proposition 3 - Under the hypotheses of Theorem A, the Patterson measure of $\Gamma$ gives full measure to the set $\Lambda^{0}$ and $\Gamma$ is divergent.

Let us recall here the construction of the Patterson measure. By [9], there exists a non 
negative function $h$ on $\mathbb{R}^{+}$such that, for any $\mathbf{x}, \mathbf{y}$ in $\mathbb{H}^{d+1}$, the series

$$
P_{\Gamma}^{\prime}(\mathbf{x}, \mathbf{y}, s)=\sum_{\gamma \in \Gamma} e^{-s(\mathbf{x}, \gamma \cdot \mathbf{y})} h((\mathbf{x}, \gamma \cdot \mathbf{y}))
$$

diverges if and only if $s \leq \delta_{\Gamma}$; if $\Gamma$ is divergent one takes $h=1$, otherwise the function $h$ is strictly increasing and satisfies the following property :

For any $\epsilon>0$, there exists $r_{\epsilon} \geq 0$ such that $\forall t \geq 0, \forall r \geq r_{\epsilon} \quad h(t+r) \leq e^{\epsilon t} h(r)$. For $s>\delta_{\Gamma}$ set $\sigma_{\mathbf{x}, \mathbf{y}, s}=\frac{1}{P^{\prime}(\mathbf{y}, \mathbf{y}, s)} \sum_{\gamma \in \Gamma} e^{-s(\mathbf{x}, \gamma \cdot \mathbf{y})} h((\mathbf{x}, \gamma \cdot \mathbf{y})) \epsilon_{\gamma \cdot \mathbf{y}}$ where $\epsilon_{\gamma \cdot \mathbf{y}}$ is the Dirac mass at $\gamma \cdot \mathbf{y}$.There exists a sequence $\left(s_{i}\right)$ in $\mathbb{R}^{+}$converging to $\delta_{\Gamma}$ from above such that $\left(\sigma_{\mathbf{x}, \mathbf{y}, s_{i}}\right)$ weakly converges to a measure $\sigma_{\mathbf{x}, \mathbf{y}}$ with support $\Lambda_{\Gamma}$; for any $\mathbf{x}^{\prime}$ in $\mathbb{H}^{d+1}$, the sequence $\left(\sigma_{\mathbf{x}^{\prime}, \mathbf{y}, s_{i}}\right)$ also weakly converges and its limit $\sigma_{\mathbf{x}^{\prime}, \mathbf{y}}$ is absolutely continuous with respect to $\sigma_{\mathbf{x}, \mathbf{y}}$ with Radon-Nikodym derivative $\frac{d \sigma_{\mathbf{x}^{\prime}, \mathbf{y}}}{d \sigma_{\mathbf{x}, \mathbf{y}}}(\xi)=e^{-\delta_{\Gamma} B_{\xi}\left(\mathbf{x}^{\prime}, \mathbf{x}\right)}$. Furthermore, for any $\gamma \in \Gamma$ one has $\gamma^{*} \sigma_{\mathbf{x}, \mathbf{y}}=\sigma_{\gamma^{-1} . \mathbf{x}, \mathbf{y}}$.

Let $\Gamma_{H}^{*}$ be the set of $\gamma \in \Gamma$ with first letter in $H^{*}$. From now on, we set $G^{*}=\left\{g_{i}, i \geq 1\right\}$ and we enlarge $F_{H}$ in such a way that $\Lambda_{\Gamma} \cap F_{H}$ is included in the interior of $F_{H}$. Consider the open set $U=\overline{H^{d+1}}-\left(\Gamma_{H}^{*} \cdot \mathbf{y} \cup F_{H}\right)$; for any $k \geq 1$, the set $U_{k}=U \cap g_{1} U \cap \cdots \cap g_{k} U$ is also open in $\overline{\mathbb{H}^{d+1}}$ and contains all the $\Gamma$-orbit of $\mathbf{y}$ but the $\gamma \cdot \mathbf{y}, g_{1} \gamma \cdot \mathbf{y}, \ldots, g_{k} \gamma \cdot \mathbf{y}$ with $\gamma \in \Gamma_{H}^{*}$. For $s>\delta_{\Gamma}$ one has $\sigma_{\mathbf{x}, \mathbf{y}, s}\left(U_{k}\right) \leq \frac{1}{P^{\prime}(\mathbf{y}, \mathbf{y}, s)} \sum_{l>k} \sum_{\gamma \in \Gamma_{H}^{*}} e^{-s\left(\mathbf{x}, g_{l} \gamma \cdot \mathbf{y}\right)} h\left(\left(\mathbf{x}, g_{l} \gamma \cdot \mathbf{y}\right)\right)$. Choose $\epsilon>0$ quite small such that $\delta_{\Gamma}>\delta_{G}+\epsilon$ and $\mathbf{x}$ in such a way that $\operatorname{dist}(\mathbf{x}, \Gamma \cdot \mathbf{y}) \geq r_{\epsilon}$ ; for $l>k$ and $\gamma \in \Gamma_{H}^{*}$ one has $h\left(\left(\mathbf{x}, g_{l} \gamma \cdot \mathbf{y}\right)\right) \leq e^{\epsilon\left(\mathbf{x}, g_{l} \cdot \mathbf{x}\right)} h((\mathbf{x}, \gamma \cdot \mathbf{y}))$. On the other hand, since the visibility angle between $F_{G}$ and $F_{H}$ is bounded from below, there exists $\theta>0$ such that the angle at $\mathbf{x}$ between the geodesic segments $\left[g^{-1} \cdot \mathbf{x}, \mathbf{x}\right]$ and $[\mathbf{x}, \gamma \cdot \mathbf{y}]$ is greater than $\theta$ for all but finitely many $g \in G^{*}$ and $\gamma \in \Gamma_{H}^{*}$; so there exists $C>0$ such that $(\mathbf{x}, g \gamma \cdot \mathbf{y}) \geq(\mathbf{x}, g \mathbf{x})+(\mathbf{x}, \gamma \cdot \mathbf{y})-C$ for any $g \in G^{*}$ and $\gamma \in \Gamma_{H}^{*}$. It readily follows $\sigma_{\mathbf{x}, \mathbf{y}, s}\left(U_{k}\right) \leq e^{s C} \sigma_{\mathbf{x}, \mathbf{y}, s}\left(\overline{\mathbb{H}^{d+1}}\right) \sum_{l>k} e^{(-s+\epsilon)\left(\mathbf{x}, g_{l} \cdot \mathbf{x}\right)}$. Letting $s \rightarrow \delta_{\Gamma}$ along the sub-sequence $\left(s_{i}\right)$ leads to

$$
\sigma_{\mathbf{x}, \mathbf{y}}\left(\Lambda_{G}\right) \leq \sigma_{\mathbf{x}, \mathbf{y}}\left(U_{k}\right) \leq e^{\delta_{\Gamma} C} \sigma_{\mathbf{x}, \mathbf{y}}\left(\mathbb{S}^{d}\right) \sum_{l>k} e^{\left(-\delta_{\Gamma}+\epsilon\right)\left(\mathbf{x}, g_{l} \cdot \mathbf{x}\right)}
$$

and the inequality $\delta_{\Gamma}-\epsilon>\delta_{G}$ implies $\sigma_{\mathbf{x}, \mathbf{y}}\left(\Lambda_{G}\right)=0$. Similarly $\sigma_{\mathbf{x}, \mathbf{y}}\left(\Lambda_{H}\right)=0$ and so $\sigma_{\mathbf{x}, \mathbf{y}}\left(\Lambda^{0}\right)=\sigma_{\mathbf{x}, \mathbf{y}}\left(\mathbb{S}^{d}\right)$. The Patterson measure thus gives full measure to the radial limit set of $\Gamma$, which implies that $\Gamma$ is divergent. Furthermore, the action of $\Gamma$ on $\mathbb{S}^{d}$ is ergodic with respect to $\sigma_{\mathbf{x}, \mathbf{y}}$ and the measure $\sigma_{\mathbf{x}, \mathbf{y}}$ does not depend neither on $\mathbf{y}$ nor on the sequence $\left(s_{i}\right)$ which appears in its construction; it will be denoted $\sigma_{\mathbf{x}}$ in the sequel. $\square$ 


\section{Proof of Theorem A and its corrolaries}

The $\Gamma$-conformality of the family $\left(\sigma_{\mathbf{x}}\right)$ implies that the measure $\frac{\sigma_{\mathbf{o}}\left(d \xi^{-}\right) \sigma_{\mathbf{o}}\left(d \xi^{+}\right)}{\left|\xi^{-}-\xi^{+}\right|^{2 \delta_{\Gamma}}}$ is a $\Gamma$ invariant Radon measure on $\Lambda_{\Gamma} \times \Lambda_{\Gamma}$-diagonal : this is the geodesic current $c^{\sigma}$ associated with $\left(\sigma_{\mathbf{x}}\right)$. The measure $\tilde{\mu}^{\sigma}=c^{\sigma} \otimes d t$ is invariant both under the actions of $\Gamma$ and of $\left(\tilde{g}_{t}\right)$, it induces on $T^{1} M$ a $\left(g_{t}\right)$-invariant measure $\mu^{\sigma}$ called the Patterson-Sullivan measure.

By Proposition 3, the set $\left(\Lambda^{0} \times \Lambda^{0}-\right.$ diagonal $) \times \mathbb{R} / \Gamma$ has full measure with respect to $\mu^{\sigma}$ and the geodesic flow $\left(g_{t}\right)$ restricted to this set can be presented as a special flow constructed from the automorphism $T$ on $\mathcal{D}^{0}$ and the ceiling function $f$.

When $f$ is strictly positive on $\mathcal{D}^{0}$, the set $\left\{\left(\xi^{-}, \xi^{+}, r\right) /\left(\xi^{-}, \xi^{+}\right) \in \mathcal{D}^{0}, 0 \leq r<f\left(\xi^{+}\right)\right\}$is a fundamental domain for the action of $T_{f}$ on $\mathcal{D}^{0} \times \mathbb{R}$. More generally, by Lemma 1 , the function $f$ is bounded from below and there exists $n_{0} \geq 1$ such that $S_{n_{0}} f=f+f \circ T+\cdots f \circ$ $T^{n_{0}-1}$ is strictly positive on $\mathcal{D}^{0}$; in particular, $f$ is semi-integrable on $\mathcal{D}^{0}$ and the sequence $\left(S_{n} f\left(\xi^{+}\right)\right)$goes to infinity on $\mathcal{D}^{0}$. By classical technics in ergodic theory, the function $f$ is cohomologous to a strictly positive function $F$ : on has $f=F+h-h \circ T$ for some measurable function $h$. The set $\mathcal{D}_{h, F}^{0}=\left\{\left(\xi^{-}, \xi^{+}, r\right) /\left(\xi^{-}, \xi^{+}\right) \in \mathcal{D}^{0}, h\left(\xi^{+}\right) \leq r<h\left(\xi^{+}\right)+F\left(\xi^{+}\right)\right\}$ is thus a fundamental domain for the action of $T_{f}$ on $\mathcal{D}^{0} \times \mathbb{R}$.

The measure $\mu^{\sigma}$ can be identified with the restriction of $c^{\sigma} \otimes d t$ to the set $\mathcal{D}_{h, F}^{0}$. ; in particular $\mu^{\sigma}\left(T^{1} M\right)=\mu^{\sigma}\left(\mathcal{D}_{h, F}^{0}\right)=\int_{\mathcal{D}^{0}} F\left(\xi^{+}\right) c^{\sigma}\left(d \xi^{-} d \xi^{+}\right)$and the measure $\mu^{\sigma}$ is finite if and only if $\int_{\mathcal{D}^{0}}\left|f\left(\xi^{+}\right)\right| c^{\sigma}\left(d \xi^{-} d \xi^{+}\right)<+\infty$.

Set $\Lambda_{G}^{0}=\Lambda_{0} \cap F_{G}$ and $\Lambda_{H}^{0}=\Lambda_{0} \cap F_{H}$; one has $\mathcal{D}^{0}=\left(\Lambda_{H}^{0} \times \Lambda_{G}^{0}\right) \cup\left(\Lambda_{G}^{0} \times \Lambda_{H}^{0}\right)$. Let us decompose $\Lambda_{H}^{0} \times \Lambda_{G}^{0}$ in the disjoint union of the sets $\Lambda_{H}^{0} \times g \cdot \Lambda_{H}^{0}$ with $g \in G^{*}$. By Lemma 1 , the quantity $B_{\xi}\left(g^{-1} \cdot \mathbf{o}, \mathbf{o}\right)-(\mathbf{o}, g \cdot \mathbf{o})$ is bounded uniformly in $g \in G^{*}$ and $\xi \in \Lambda_{H}^{0}$ and so $\sigma_{\mathbf{o}}\left(g \cdot \Lambda_{H}^{0}\right)=\int_{\Lambda_{H}^{0}} e^{-\delta_{\Gamma} B_{\xi}\left(g^{-1} \cdot \mathbf{o}, \mathbf{o}\right)} \sigma(d \xi) \asymp e^{-\delta_{\Gamma}(\mathbf{o}, g \cdot \mathbf{o})}$ (where $a \asymp b$ means that $1 / K \leq \frac{a}{b} \leq K$ for some constant $K>1$ ). It follows

$$
\begin{aligned}
\int_{\Lambda_{H}^{0} \times \Lambda_{G}^{0}}\left|f\left(\xi^{+}\right)\right| c^{\sigma}\left(d \xi^{-} d \xi^{+}\right) & =\sum_{g \in G^{*}} \int_{\Lambda_{H}^{0} \times g \cdot \Lambda_{H}^{0}}\left|B_{\xi^{+}}(\mathbf{o}, g \cdot \mathbf{o})\right| \frac{\sigma_{\mathbf{o}}\left(d \xi^{-}\right) \sigma_{\mathbf{o}}\left(d \xi^{+}\right)}{\left|\xi^{-}-\xi^{+}\right|^{2 \delta_{\Gamma}}} \\
& \asymp \sum_{g \in G^{*}} d(\mathbf{o}, g \cdot \mathbf{o}) e^{-\delta_{\Gamma}(\mathbf{o}, g \cdot \mathbf{o})}
\end{aligned}
$$

the last estimate using the fact that the euclidean distance between the sets $F_{G}$ and $F_{H}$ is strictly positive. A similar estimate holds for $\int_{\Lambda_{G}^{0} \times \Lambda_{H}^{0}}\left|f\left(\xi^{+}\right)\right| c^{\sigma}\left(d \xi^{-} d \xi^{+}\right)$; the inequality $\delta_{\Gamma}>\max \left(\delta_{G}, \delta_{H}\right)$ implies that these integrals are finite.

Remark 2- The hypothesis $\delta_{\Gamma}>\max \left(\delta_{G}, \delta_{H}\right)$ is crucial in the proof of Theorem A. For instance, let $\langle a, b\rangle$ be a classical Schottky group generated by 2 hyperbolic isometries and let $\Gamma$ be the group generated by $\left\{a^{-n} b a^{n} / n \in \mathbb{Z}\right\}$. One has $a \notin \Gamma$ but $a \Gamma a^{-1}=\Gamma$, which implies $a^{*} \sigma_{\mathbf{o}}=\sigma_{a^{-1} \cdot \mathbf{o}}$; so the Patterson-Sullivan measure $\mu^{\sigma}$ on $T^{1}\left(I^{d+1} / \Gamma\right)$ is infinite since it is invariant under the action of $a$. Let us now check that the hypothesis 
$\delta_{\Gamma}>\max \left(\delta_{G}, \delta_{H}\right)$ is not satisfied, whatever decomposition $\Gamma=G * H$ with $G$ and $H$ in Schottky position one takes. If $a^{n} b a^{-n} \in G$ then $\Lambda_{G}$ contains $a^{n} \xi_{b}$ where $\xi_{b} \in \mathbb{S}^{d}$ is fixed by $b$; the fact that $G$ and $H$ are in Schottky position implies that one of the two subgroups, say $G$, contains all the $a^{n} b a^{-n}$ for $n$ large enough. So $\Gamma=\bigcup_{k \geq 0} a^{-k} G a^{k}$ and since $\delta_{a^{-k} G a^{k}}=\delta_{G}$ it follows $\delta_{\Gamma}=\lim _{k \rightarrow+\infty} \delta_{a^{-k} G a^{k}}=\delta_{G}$ (note also that $G$ is of convergent type by Proposition 2 in [5]).

Proof of Corollary 1- Its suffices to find an infinitely generated classical Schottky group which can be decomposed in a Schottky product satisfying the hypotheses of Theorem A. Let $\langle\alpha, \beta, a, b\rangle$ be a classical Schottky group generated by 4 hyperbolic isometries and assume that the critical exponent of $\langle\alpha, \beta\rangle$ is greater than the one of $<a, b\rangle$. Set $G=<\alpha, \beta\rangle$ and consider the sub-group $H$ of $\langle a, b\rangle$ generated by $\ldots a^{-2} b a^{2}, a^{-1} b a^{1}, b, a b a^{-1}, a^{2} b a^{-2} \ldots$; one has $\delta_{H} \leq \delta_{G}$. Since $G$ is convex-cocompact, it is divergent, and the remark 1 allows us to conclude.

To prove the second assertion, will use the following formula due to Th. Roblin [11] :

$$
\int_{N^{\epsilon}(\Gamma)} \Phi_{\sigma}(x)^{2} d V_{\Gamma}(x)=\mu^{\sigma}\left(T^{1} M\right) \int_{v \in \mathbb{R}^{d}} \frac{1_{\left[0, s i n h^{-1}(\epsilon)\right]}(\|v\|)}{\left(1+\|v\|^{2}\right)^{\delta_{\Gamma}}} d v
$$

where $d V_{\Gamma}$ denotes the volume form on $\mathbb{B}^{d+1} / \Gamma$. If $\alpha, \beta, a, b$ are such that the critical exponent of the Schottky group they generate is $\leq d / 2$, one has $\int_{N^{\epsilon}(\Gamma)} \Phi_{\sigma}(x)^{2} d V_{\Gamma}(x)<+\infty$ for any $\epsilon>0$. On the other hand, if one replaces the group $G=<\alpha, \beta>$ by a parabolic group of rank $d$, one has $\delta_{\Gamma}>d / 2$ and the Patterson-Sullivan measure of $\Gamma=G * H$ is finite. Consequently $\int_{v \in \mathbb{R}^{d}} \frac{d v}{\left(1+\|v\|^{2}\right)^{\delta_{\Gamma}}}<+\infty$ and so $\int_{\mathbb{H}^{d+1} / \Gamma} \Phi_{\sigma}(x)^{2} d V_{\Gamma}(x)<+\infty$ by the previous formula.

Proof of Corollary 2- Consider finitely many Kleinian transformations $\alpha_{1}, \cdots, \alpha_{N}$ which generate a non geometrically finite group in $\operatorname{PSL}(2, \mathbb{C})$ and let $G$ be the group of isometries of $\mathbb{H}^{d+1}$ generated by the Poincaré extension of $\alpha_{1}, \cdots, \alpha_{N}$ on $\mathbb{H}^{d+1}$; one has $\delta_{G} \leq 2$ and one may choose a closed set $F_{G} \subset \mathbb{S}^{d}$ such that $g\left(\mathbb{S}^{d}-F_{G}\right) \subset F_{G}$ for any $g \in G^{*}$. Let $H_{0}$ be a divergent group of finite type of isometries of $\mathbb{H}^{d+1}$, whose limit set is included in $F_{G}$ and such that $\delta_{H_{0}}>2$. At last consider an hyperbolic isometry $\alpha$ whose fixed points belong to $\mathbb{S}^{d}-F_{G}$; for $n$ large enough there exists a closed set $F_{H} \subset \mathbb{S}^{d}-F_{G}$ such that the group $H=\alpha^{-n} H_{0} \alpha^{n}$ maps the exterior of $F_{H}$ in its interior. In other words, $G$ and $H$ are in Schottky position. Furthermore, the divergence of $H$ implies $\delta_{G * H}>\delta_{H}=\max \left(\delta_{G}, \delta_{H}\right)$. The group $G * H$ is finitely generated, it is not geometrically finite by theorem $\mathrm{C} 2$ (xi) in [8] (with the same proof in every dimension) and its Patterson-Sullivan measure is finite by Theorem A. 


\section{References}

[1] Ancona A. Exemples de surfaces hyperboliques de type divergent, de mesure de Sullivan associées finies mais non géométriquement finies, Preprint

[2] Bers L. On boundaries of Teichmüller spaces and on Kleinian groups, Ann. of Math. 91, (1970), p. 570-600.

[3] Bishop C.J. \& Jones P.W. Hausdorff dimension and kleinian groups, Acta Math. 179, (1997), p. 1-39.

[4] Bowditch B. Geometrical finiteness with variable negative curvature, Duke Math. J. vol. 77, (1995), p. 229-274.

[5] Dal'bo F., \& Otal J.P. \& Peigné M. Séries de Poincaré des groupes géométriquement finis, Israel Jour. Math. 118, (2000), 109-124.

[6] Furusawa H. Poincaré series of combination groups, Tôhoku Math. J. 43 (1991), p. 1-7.

[7] Hamilton E. Geometrical finiteness for hyperbolic orbifolds, Topology 37, n. 3, (1998), p. 635-657.

[8] Maskit B. Kleinian groups, A series of comprehensive Studies in Mathematics, Springer Verlag (1987)

[9] Patterson S.J. The limit set of a Fuchsian group, Acta Math. 136, (1976), p. 241-273.

[10] Patterson S.J. The exponent of convergence of Poincaré series, Monats. für Math. 82 (1976), p. 297-315.

[11] Roblin Th. Sur la théorie ergodique des groupes discrets en géométrie hyperbolique, Thèse de doctorat de l'Université d'Orsay (1999)

[12] Sullivan D. The density at infinity of a discrete group of hyperbolic motions, Publ. Math. IHES, vol. 50, (1979), p. 171-202.

[13] Sullivan D. Entropy, Hausdorff measures old and new, and limit sets of geometrically finite Kleinian groups, Acta Math. 153, (1984), p. 259-277.

[14] Sullivan D. Discrete conformal groups and measurable dynamics, Bull. A.M.S., vol.6, $n^{\circ} 1,(1984)$, p. 259-277. 